\title{
Functions of Cancer-Derived Extracellular Vesicles in Immunosuppression
}

\author{
Liliana Czernek $^{1} \cdot$ Markus Düchler $^{1}$
}

Received: 14 July 2016 / Accepted: 18 December 2016 / Published online: 18 January 2017

(C) The Author(s) 2017. This article is published with open access at Springerlink.com

\begin{abstract}
Extracellular vesicles, including exosomes, constitute an important element of intercellular communication by carrying a variety of molecules from producer to target cells. The transport of mRNA and miRNA can directly modulate gene expression in the target cells. The miRNA content in exosomes is characteristic for the cell from which the vesicles were derived enabling the usage of exosomes as biomarkers for the diagnosis various diseases, including cancer. Cancer-derived exosomes support the survival and progression of tumors in many ways and also contribute to the neutralization of the anti-cancer immune response. Exosomes participate in all known mechanisms by which cancer evades the immune system. They influence the differentiation and activation of immune suppressor cells, they modulate antigen presentation, and are able to induce T-cell apoptosis. Although cancer-derived exosomes mainly suppress the immune system and facilitate tumor progression, they are also important sources of tumor antigens with potential clinical application in stimulating immune responses. This review summarizes how exosomes assist cancer to escape immune recognition and to acquire control over the immune system.
\end{abstract}

Keywords Exosomes $\cdot$ Extracellular vesicles $\cdot$ Cancer immunosuppression $\cdot$ Suppressor cells $\cdot$ Immune escape

Markus Düchler

mduchler@cbmm.lodz.pl

1 Department of Bioorganic Chemistry, Centre of Molecular and Macromolecular Studies, Polish Academy of Sciences, Sienkiewicza 112, 90-363 Lodz, Poland

\section{Introduction}

In the early 1980s, two research groups described that the transferrin receptor of sheep reticulocytes was secreted via little-known vesicular forms (Pan and Johnstone 1984). The same researchers described the mechanism of small vesicle secretion showing that the release of membrane vesicles was preceded by inward budding of an intracellular endosome forming a multi-vesicular body (MVB), which could then fuse with the plasma membrane (Pan et al. 1985). Rose Johnstone used the term "exosomes" for the first time to describe small membrane vesicles formed in MVBs (Johnstone et al. 1987). The original function attributed to membrane vesicles was the removal of cell debris. The thinking about membrane vesicles as "trash cans" of the cell was derived from the knowledge about the role of lysosomes as degradation centers (Luzio et al. 2007). Since the finding that exosomes can modulate the immune system, extracellular vesicles gained growing interest (Raposo et al. 1996). The enthusiasm was further increased after the discovery of mRNA and miRNA inside exosomes (Valadi et al. 2007). These studies opened the door to the new research field of exosome functions in intercellular communication, their biomarkers, and their potential role as therapeutic tools.

\section{Classification of Extracellular Vesicles}

Cells release different kinds of extracellular vesicles (EVs) of varying sizes and biogenesis. Their classification distinguishes three main subpopulations/classes based on the vesicle's origin. The smallest vesicles are of endocytic origin, exosomes, with $40-150 \mathrm{~nm}$ in diameter (Baietti et al. 2012; Colombo et al. 2013). Ectosomes, also called 
shedding microvesicles, with a diameter of $100-1000 \mathrm{~nm}$ are produced by outward protrusion or budding from the plasma membrane (Muralidharan-Chari et al. 2009; Théry et al. 2009). The most heterogeneous group of vesicles ranging from 50 up to $5000 \mathrm{~nm}$ in diameter is apoptotic bodies. Their biogenesis is based on fragmentation of apoptotic cells during programmed cell death (Mathivanan et al. 2010; Théry et al. 2009). A common feature of all vesicle classes is their membrane structure, a lipid bilayer with the same topological orientation as the plasma membrane (Trajkovic et al. 2008). Although the origin of microvesicles and exosomes is well known, the experimental discrimination of these vesicles types is difficult, and so the terms are sometimes subsumed as extracellular vesicles. In this review, we follow the terminology used by the authors.

\section{Exosome Biogenesis}

The process of exosome biogenesis is not fully understood. It starts within endosomes which are responsible for regulated trafficking of proteins and lipids between subcellular compartments of the secretory and endocytic pathway (Lemmon and Traub 2000). The cargo of endosomes can enter recycling circuits to return membrane components back to the plasma membrane, or can be sorted into lysosomes for degradation (Huotari and Helenius 2011). The content of cholesterol is associated with the fate of MVBs; cholesterol-poor MVBs are appointed for lysosome fusion and degradation (Möbius et al. 2002). Exosomes formed within MVBs are released via exocytosis into the extracellular space when cholesterol-rich MVBs fuse with the plasma membrane (Kalra et al. 2012).

During vesicle formation, cellular components, extracellular ligands, and other endocytosed molecules, such as receptors, are packed into the vesicles (Gould and Lippincott-Schwartz 2009). Molecules from the early endosomes, such as the tetraspanin CD63, or LAMP1 and LAMP2, are released through the vesicles (Colombo et al. 2014; Jaiswal et al. 2002; Raposo et al. 1996). The ESCRT (endosomal sorting complex required for transport) machinery is involved in the budding process, as well as in the controlled sorting of proteins into exosomes. The ESCRT machinery consists of four complexes, ESCRT-0 (Hrs), ESCRT-I (TSG101 and Vps28), ESCRT-II (Vps22), and ESCRT-III (Alix and Vps2), which sort ubiquitinylated proteins to the late endosomes. The ESCRT-III complex was shown to promote intraluminal budding of vesicles in endosomes which results in maturation of the cargo-containing vesicles (Colombo et al. 2013; Kowal et al. 2014). An ESCRT-independent packaging mechanism was also proposed involving glycolipoprotein microdomains (lipid rafts) (Trajkovic et al. 2008). Besides a big range of proteins, also nucleic acids like mRNA, miRNA, or DNA can be found in exosomes (http://www.exocarta.org) (Thakur et al. 2014; Valadi et al. 2007). Interestingly, the miRNA contents of exosomes do not entirely parallel the miRNA composition inside the cell indicating selective loading mechanisms (Rappa et al. 2013). For the selection of miRNA for exosomal export, several potential routes were described, one depending on neural sphingomyelinase 2 (Kosaka et al. 2013), a second based on uridylation versus adenylation of the 3 'end of the miRNAs (Koppers-Lalic et al. 2014), a third one involving sumoylated heterogeneous nuclear ribonuleoprotein (hnRNPA2B1) binding to a GGAG motif in the 3'part of miRNA sequences to be packed into exosomes (VillarroyaBeltri et al. 2013), and another one related to the RISC pathway (Gibbings et al. 2009). For the selective loading of mRNA into microvesicles, a 25-nucleotide sequence motif in the $3^{\prime}$-UTR of exported mRNAs was described (Bolukbasi et al. 2012). In cancer cell-derived exosomes, also fragments of chromosomal DNA were identified (Kahlert et al. 2014), their sorting mechanism into the vesicles has not yet been defined. The analysis of the miRNA content of exosomes allows to draw conclusions about the cell type from which the exosomes originated. Thus, determining the miRNA profile in extracellular vesicles derived from bodily fluids of diseased persons has a huge potential for diagnostic purposes (Miller and Grunewald 2015; Verma et al. 2015).

\section{Cancer's Immune Escape}

The immune system provides a defense against attacks of foreign invaders, such as bacteria, viruses, and parasites, or the growth of cancer cells. Once it recognizes non-self antigens, it activates multiple chemical and physiological processes constituting the immune response (Kindt et al. 2007). The immune response comprises innate and adaptive immunity. The components of the innate response include antigen-presenting cells (APCs) like macrophages or dendritic cells (DCs) that are responsible for phagocytosis, digestion, and presentation of pathogen-derived antigens on the cell surface, and natural killer (NK) cells that directly destroy infected or transformed cells. The innate immune response is followed by the adaptive one which is based on activation of specific $\mathrm{B}$ and $\mathrm{T}$ lymphocytes. $\mathrm{T}$ cells are highly specialized cells that not only coordinate (T-helper: Th) or suppress (T-regulatory: Treg) the immune response, but also destroy infected cells (T-cytotoxic: CTL). B cells secrete antibodies which mark infected cells or pathogens to promote their elimination from the organism. The T-cell and B-cell responses include the production of memory cells against the pathogen enabling quicker immune response in future challenges (Kindt et al. 2007). 
Cancer cells have to express antigens which are recognized as non-self to elicit an immune response. Such tumor-associated antigens (TAA) are either mutated cellular proteins, or molecules with differences in posttranslational modifications (Finn 2012). TAA-derived peptides produced by the proteasome are presented through major histocompatibility complex (MHC) I complexes on the cell surface and recognized by CTLs resulting in tumor cell killing. The strategies used by tumors to escape this destruction include the impairment of the executory capacity of the immune system, and hiding from recognition by immune cells through the loss of target antigen expression. Defective antigen presentation can be caused by the down-regulation of the antigen processing machinery which may affect the MHC-I pathway and other involved proteins like the proteasome subunits LMP2 (latent membrane protein 2) and LMP7, the transporter associated with antigen processing, and tapasin (Garrido et al. 1997; Hicklin et al. 1999; Johnsen et al. 1999; Restifo et al. 1993; Rotem-Yehudar et al. 1996). When the expression of TAA is down-regulated, CTL no longer recognize the tumor cells (Maeurer et al. 1996). About 20 years ago, mutations in the $\beta 2$-microglobulin gene have been identified in metastatic melanoma cells resulting in the absence of HLA class I antigens on the cell surface (Benitez et al. 1998).

The production of immune suppressive cytokines by cancer cells or non-cancer cells in the tumor microenvironment exerts a powerful suppression of the anti-cancer immune response. Among these cytokines are transforming growth factor (TGF)- $\beta$, tumor necrosis factor (TNF)- $\alpha$, interleukin (IL)-1, IL-6, IL-8, IL-10, and type I interferons (IFNs) (Pasche 2001; Lind et al. 2004; Matsuda et al. 1994). Furthermore, vascular endothelial growth factor (VEGF) has the ability to suppress proper T-cell development and function (Ohm et al. 2003). TGF- $\beta$ and IL-10 can shift the balance from a Th1 response executed by cytotoxic $\mathrm{T}$ cells towards an antibody-based Th2 response (immune deviation) (Maeda and Shiraishi 1996). Induction of immune tolerance may also occur through down-regulation of co-stimulatory molecules on APCs. Engagement of the T-cell receptor (TCR) in the absence of co-stimulation induces anergy or tolerance in T cells (Staveley-O'Carrol et al. 1998). Tumors even eliminate tumor-specific CTLs by expressing ligands to death receptors which trigger T-cell apoptosis (Bogen 1996). Advanced cancer-induced immunosuppression results in the induction and activation of immune suppressor cells like myeloid-derived suppressor cells (MDSCs) and Treg cells. Treg cells generally suppress the activity and proliferation of effector $\mathrm{T}$ cells (Shevach 2002), fulfil an important function to maintain immune tolerance to self-antigen, and are critical in the suppression of autoimmune diseases. It was shown that tumor-derived Tregs have comparatively higher suppressive activity than naturally occurring Tregs (Yokokaw et al. 2008; Gasparoto et al. 2010). The induction and activation of cancer-antigen-specific Treg cells seem to be the major mechanism of tumor immune escape (Vinay et al. 2015).

\section{Specific Features of Cancer Cell-Derived Extracellular Vesicles}

Cancer cells release increased amounts of exosomes compared to their non-transformed counterparts (Pap et al. 2011). Chemotherapy or photo-dynamic treatment further boosts the release of extracellular vesicles (Aubertin et al. 2016). Cancer-derived exosomes were shown to contribute to tumor angiogenesis, to transport growth promoting proteins, such as mutant KRAS, epidermal growth factor receptor (EGFR), and SRC family kinases, to induce therapy resistance by removal of chemotherapeutic drugs, and to prepare metastatic niches for the colonization of circulating cancer cells (reviewed in Miller and Grunewald 2015). For example, Al-Nedawi et al. (2008, 2009) showed that exosomes transferred functional EGFR from cancer cells to endothelial cells. Subsequently, VEGF secretion was induced which triggered autocrine VEGF signaling by binding to the endothelial VEGFR-2 and resulted in neovascularization.

Due to their complex structure, exosomes may contribute to both, stimulation and suppression of immune responses. Whether cancer-derived exosomes stimulate immunity or tolerance seems to also depend on the amount of transferred vesicles with a suppressive effect at high vesicle concentration (Hellwinkel et al. 2016).

\section{Immune Stimulation}

Exosomes can promote immune responses by regulating signals for both, adaptive and innate immune responses (Zhang et al. 2014). Cancer exosomes bear MHC class I and class II complexes at their surface and were able to function as antigen-presenting vesicles to directly activate T cells (Raposo et al. 1996). Exosomes derived from both human and murine B lymphocytes induced antigen-specific MHC class II-restricted T-cell responses. Primed antigenspecific $\mathrm{T}$ cells were efficiently stimulated by MHC II complexes on exosomes secreted from activated B cells, suggesting a role for B-cell-derived exosomes to modulate an ongoing immune response or to maintain antigen-specific memory $\mathrm{T}$ cells. However, T-cell priming was necessary, as B-cell-derived exosomes could stimulate primed $\mathrm{CD}^{+}$ T cells, but not naïve T cells (Muntasell et al. 2007; Raposo et al. 1996). 
In an indirect, but more efficient pathway of immunostimulation, exosomes transfer tumor antigens to DCs and other APCs (Denzer et al. 2000; Morelli et al. 2004; Wolfers et al. 2001). Tumor antigens from cancer-derived exosomes are captured and presented by APCs to induce efficient anti-tumor immune responses (Rao et al. 2016). This pathway has extensively been exploited for cancer vaccination and immunization with exosomes isolated from ascites in colorectal cancer patients who were explored in phase I clinical trials (Dai et al. 2008). Human DCs loaded with glioma-derived exosomes activated a tumor-specific CTL response in vivo ( $\mathrm{Bu}$ et al. 2011). Exosome treatment stimulated the up-regulation of MHC II molecules and the co-stimulatory receptors CD80 and CD86 on the DCs. The efficiency of anti-tumor immunity induction could be increased by either stimulation of Rab27a over-expression to boost exosome secretion ( $\mathrm{Li}$ et al. 2013), or by expressing cytokines, such as TNF- $\alpha$, in the exosome producing cells (Xie et al. 2010). DCs loaded with tumor-derived exosomes were more efficient in vaccination of mice than DCs loaded with tumor cell lysates (Gu et al. 2015; Yao et al. 2013). Furthermore, targeting of tumor antigens to exosomes improved the vaccination efficacy (Rountree et al. 2011). Another successful vaccination approach employed exosomes loaded with tumor peptide antigen and $\alpha$-galactosylceramide to specifically target and activate NKT cells (Gehrmann et al. 2013). Increased levels of heatshock proteins on exosomes derived from heat-shocked lymphoma cells improved the anti-tumor immune response (Chen et al. 2006). The presentation of tumor antigens to DCs by exosomes could also be improved by modification of exosomes with immunostimulatory CpG DNA (Morishita et al. 2016).

Dendritic cells not only take up, but also produce vesicles to transfer $\mathrm{MHC} /$ peptide complexes to other immune cells (André et al. 2004). Such exosomes derived from TAA-loaded DCs were directly used as cancer vaccines (Escudier et al. 2005; Mahaweni et al. 2013; Näslund et al. 2013; Viaud et al. 2010). Théry et al. (2002) showed that DC-derived exosomes could stimulate naïve $\mathrm{CD}^{+} \mathrm{T}$ cells in vivo. Incorporation of poly(I:C), a ligand for Toll-like receptor 3 (TLR3), into antigen-loaded exosomes was demonstrated to improve the vaccination efficiency of DCderived vesicles (Damo et al. 2015). This approach could be further improved using exosomes derived from mature DCs pulsed with DC-derived exosomes (Hao et al. 2007). The usage of DC-derived exosomes for cancer vaccination has reached phase I clinical trials which demonstrated the safety of this approach (Escudier et al. 2005).

Pro-inflammatory effects of tumor-derived exosomes were described for macrophages. Exosomes derived from melanoma cells affected the cytokine and chemokine profile in macrophages (Marton et al. 2012). Wu et al. (2016) demonstrated that macrophages activated by gastric cancerderived exosomes acquired a pro-inflammatory phenotype. Exosome uptake by macrophages stimulated the NF- $\kappa \mathrm{B}$ pathway to increase the expression of pro-inflammatory factors, such as IL-6 and TNF- $\alpha$ (Wu et al. 2016). A similar NF- $\kappa \mathrm{B}$-dependent up-regulation of inflammatory factors via TLR2 was found by Chow et al. (2014). Interestingly, in this study, the inflammatory response was elicited only by exosomes secreted by breast cancer cells but not by vesicles from non-cancerous cell lines.

In conclusion, exosomes derived from cancer cells or TAA-loaded APCs provide a promising tool for cancer immunotherapy and vaccination due to their immunogenicity which can be further increased by innovative approaches.

\section{The Functions of Cancer-Derived Exosomes in Immunosuppression}

To escape destruction by the immune response, tumors avoid to be recognized by cytotoxic cells, directly impair the functioning of APCs or cytotoxic cells, or induce suppressor cells which consequently shut down immune reactions. Immune cells are even converted into supporters of tumor growth and survival. Exosomes participate in all these strategies through proteins exposed at their surface, and intra-vesicular cytokines and nucleic acids (Fig. 1).

\section{Defective Antigen Presentation}

The body's own cells are protected from the attack of cytotoxic $\mathrm{T}$ cells by exposing on their surface MHC class I molecules presenting peptides derived from unmutated normal proteins. Tumor cells expressing MHC-I/ TAA-peptide complexes instead are destroyed by cytotoxic $\mathrm{T}$ cells. To escape this destruction, cancer cells may down-regulate MHC-I expression. However, according to the "missing self" hypothesis, cells lacking MHC-I/ self-peptide expression ("missing self") are recognized and destroyed by NK cells (Ljunggren and Kärre 1990). In this way, the immune system counteracts the escape strategy of transformed and virus infected cells through down-regulation of MHC-I. To avoid destruction by NK cells after shutting down MHC-I expression, cancer cells have to find a way to inhibit NK-cell cytotoxicity. One possibility is the shedding of exosomes which affect the cytotoxic ability of NK cells (Clayton et al. 2008). NK-cell activity is regulated by the interplay of activating and inhibitory receptors. One of the activating receptors is NKG2D (NK group 2, member D) which interacts with its human ligands MIC-A and MIC-B (MHC class I chain-related proteins A and B) and ULBP 
Fig. 1 Schematic representation of the most important functions of cancer-derived exosomes in immunosuppression. Active molecules transported by exosomes and their effects on immune cells are indicated

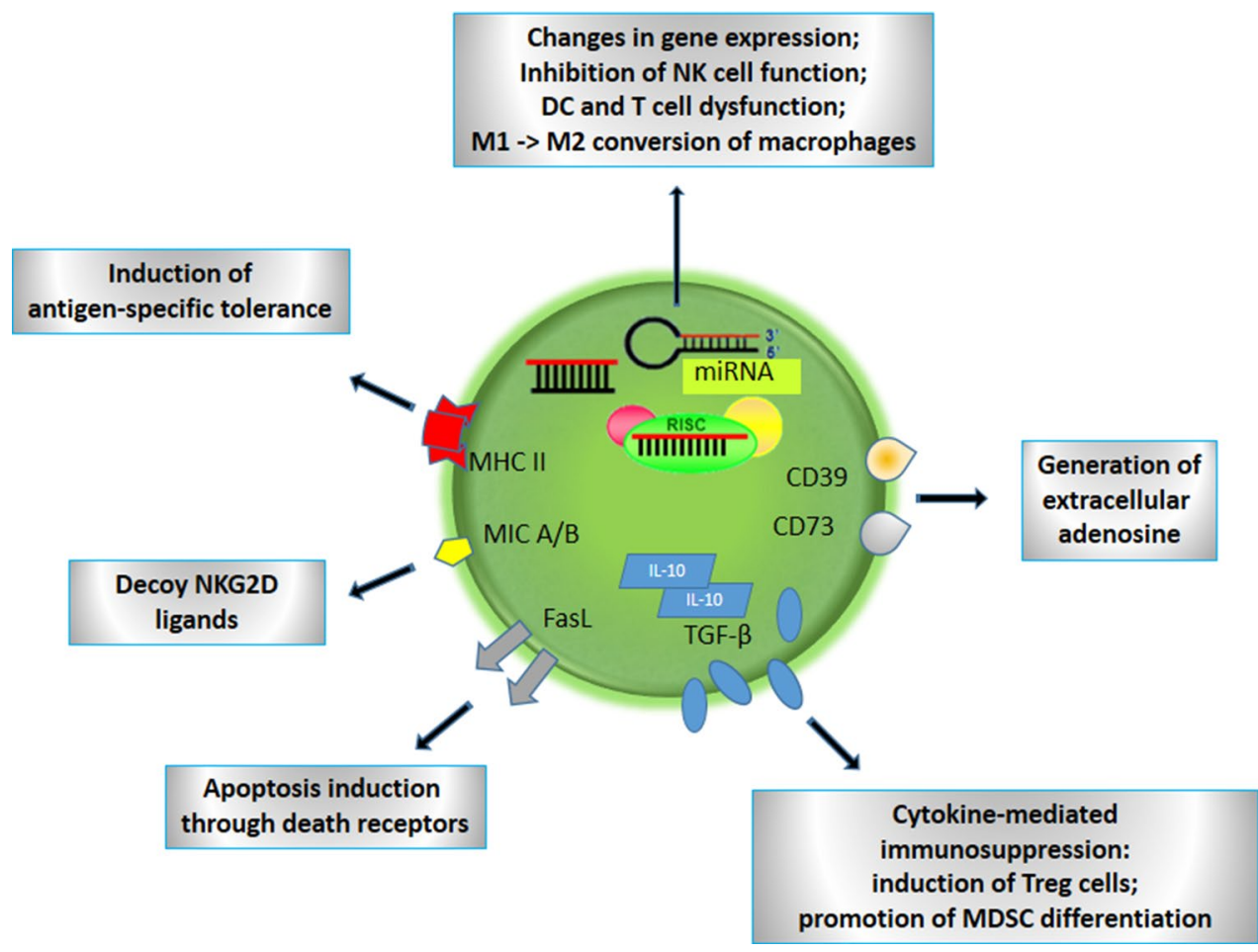

(UL-16-binding protein) (Groh et al. 2002; Raulet and Guerra 2009). Hedlund et al. (2011) showed that NKG2D ligands (MIC-A/B and ULBP 1 and 2) are expressed and secreted on exosomes. The authors demonstrated that NKG2D ligand-carrying exosomes impair NKG2D-mediated NK-cell cytotoxicity by acting as a decoy and, thus, contribute to the immune evasion of leukemia/lymphoma cells (Hedlund et al. 2011). Exosomes exposing NKG2D ligands are further able to down-regulate NKG2D expression on NK cells. This was shown for exosomes produced by human prostate cancer cells (Lundholm et al. 2014) and by acute myeloid leukemia blasts (Hong et al. 2014).

\section{Suppression of APCs and Cytotoxic T Cells}

Exosomes carry a spectrum of membrane-bound factors which have been shown to mediate immune suppression, representing another mechanism utilized by tumors to evade anti-tumor functions of immune cells (Schuler et al. 2014). The prime target of direct immunosuppression is the cytotoxic $\mathrm{T}$ cell. Growth inhibition of $\mathrm{CD} 8^{+}$ cytotoxic $\mathrm{T}$ cells mediated by glioblastoma derived exosomes was shown to promote tumor growth in mice (Liu et al. 2013). A similar observation was made with microvesicles isolated from the sera of head and neck cancer and melanoma patients, which impaired signaling and proliferation of $\mathrm{CD}^{+}$CTLs (Wieckowski et al. 2009).

\section{Vesicle-Associated Immunosuppressive Cytokines}

One of the major immunosuppressive cytokines is TGF- $\beta$ which can be associated with and exposed at the exosome surface. The potency of this vesicular form to influence cell differentiation exceeds that of the soluble form (Webber et al. 2015). In acute myeloid leukemia, NK cells became suppressed by tumor-derived microvesicles via TGF- $\beta 1$ on the exosome surface (Szczepanski et al. 2011), and breast cancer-derived exosomes suppressed T-cell proliferation through this cytokine (Rong et al. 2016).

\section{Apoptosis Induction}

Tumor-derived microvesicles were shown to induce T-cell apoptosis through the receptor-mediated pathway (Taylor et al. 2003; Wieckowski et al. 2009). For example, Fas ligand (FasL)-containing microvesicles from melanoma cells triggered apoptosis of Jurkat and other lymphoid cells. Engagement of the death receptor Fas by FasL resulted in apoptotic cell death, mediated by caspase activation (Andreola et al. 2002). Exosome-like particles derived from human colorectal cancer cells expressed FasL and TNF- $\alpha$ and triggered T-cell apoptosis in vitro and in vivo (Huber et al. 2005). In addition, exosomes produced by prostate cancer cells or human B-cell-derived lymphoblastoid cell lines suppressed T-cell responses through FasL-mediated induction of apoptosis (Abusamra et al. 2005; Klinker et al. 2014). Another pathway of apoptosis induction in $\mathrm{CD}^{+}$ 
Th1 cells was described for exosomes derived from nasopharyngeal carcinoma cell. These exosomes carried high amounts of galectin- 9 which triggered cell death by binding to its cellular receptor, Tim-3 (Klibi et al. 2009).

\section{Gene Regulatory Effects}

In general, exosomes can modify the transcriptional profile of the recipient cells by receptor activation, or by directly changing gene expression through delivered nucleic acids (Skog et al. 2008; Valadi et al. 2007). In a recent study, Muller et al. (2016) showed that signals delivered by cancer exosomes induced changes in the transcriptional profile of $\mathrm{T}$ cells and that immune response-regulating genes were preferentially targeted in $\mathrm{T}$ lymphocytes, especially in activated $\mathrm{T}$ lymphocytes. Cancer exosomes co-incubated with human $\mathrm{CD}^{+}{ }^{+} \mathrm{CD} 39^{+}$Treg cells, conventional $\mathrm{CD} 4^{+}$ $\mathrm{T}$ cells, or $\mathrm{CD}^{+} \mathrm{T}$ lymphocytes differentially regulated the expression of key immune function-related genes. The changes in mRNA expression levels were dependent on the cell type and the activation status. Incubation with cancerderived exosomes increased the levels of critical immune inhibitory proteins, such as TGF- $\beta$, IL-10, COX-2, CD39, and CD73 (Muller et al. 2016).

While a role of exosomal mRNAs in cancer-mediated immunosuppression was not yet described, a few publications found an influence of exosome transported miRNAs. Ding et al. (2015) found increased levels of nine miRNAs in DCs treated with exosomes isolated from pancreatic cancer cells. Consequently, more than 200 mRNAs were down-regulated. They further demonstrated that miR-212 caused a decrease in MHC II expression by targeting regulatory factor $\mathrm{X}$-associated protein, an important transcription factor for MHC II. In exosomes from nasopharyngeal carcinoma cells, five over-expressed miRNAs (hsa-miR24-3p, hsa-miR-891a, hsa-miR-106a-5p, hsa-miR-20a-5p, and hsa-miR-1908) were identified which reduced MAPkinase signaling in $\mathrm{T}$ cells thus altering proliferation and differentiation behavior (Ye et al. 2014). Recently, it was shown that hypoxia changed the immunosuppressive potential of extracellular vesicles. Microvesicles isolated from hypoxic lung carcinoma cells showed a stronger inhibition of NK-cell function than those isolated in normoxic conditions. The immunosuppressive effect was mediated by miR-23a, in addition to TGF- $\beta$ (Berchem et al. 2015). MiR-4498 showed higher levels in hypoxic exosomes isolated from melanoma cells (own unpublished results) and might influence immune responses by targeting CD83, an immunostimulatory molecule critical for the activation of $\mathrm{T}$ cells (Su et al. 2016). In murine tumor models, mir-494 was shown to regulate the activity of MDSC (myeloid-derived suppressor cells), a major type of immunosuppressive cells (Liu et al. 2012).
Other Exosome-Triggered Direct Immunosuppressive Mechanisms

Additional mediators involved in immune suppression include CD39 and CD73 present on the surface of cancerderived exosomes (Schuler et al. 2014; Smyth et al. 2013). CD39 and CD73 initiate an ectonucleotidase cascade that generates extracellular adenosine, which has suppressive effects on T cells. It is known that adenosine in the extracellular environment is a potent immune regulatory factor protecting cells and tissues from excessive immune-mediated damage and negatively regulates local immune responses. Exosomes secreted by cancer cells contributed to extracellular adenosine production and hence indirectly modulated immune effector cells (Clayton et al. 2011). An entirely different mechanism was described for melanoma-derived exosomes which raised ROS levels in T cells resulting in impaired TCR signaling due to zeta-chain inactivation (Söderberg et al. 2007).

Tumor exosomes also exerted a direct influence on mesenchymal stem cells (MSCs). MSCs are multipotent stromal cells with important function in tissue regeneration. MSCs support cancer progression and may create a local immunosuppressive microenvironment. Lung tumor cell A549-derived exosomes induced a pro-inflammatory phenotype of MSCs. Hsp70 on the surface of the exosomes triggered signaling through TLR2 leading to activation of NF- $\mathrm{KB}$ and elevated secretion of IL-6, IL-8 and monocyte chemotactic protein 1 by MSCs ( $\mathrm{Li}$ et al. 2016). A summary of immunosuppressive effects elicited by cancerderived exosomes is presented in Table 1.

\section{Induction and Activation of Immunosuppressive Cells}

Tumor-derived exosomes were found to direct the differentiation of naïve immune cells towards an immunosuppressive phenotype and to activate the suppressor cells. The generation, expansion, and activation of Treg cells can be driven by cancer-derived exosomes (Szajnik et al. 2010; Wieckowski et al. 2009). Clayton et al. investigated that whether tumor-derived exosomes could modify lymphocyte IL-2 responses. Mesothelioma-derived exosomes induced human Treg cells $\left(\mathrm{CD} 4^{+} \mathrm{CD} 25^{+} \mathrm{Foxp} 3^{+}\right)$which exerted dominant anti-proliferative effects on other $\mathrm{T}$ and NK lymphocytes in response to IL-2. Due to an exosomerelated mechanism, IL-2 responsiveness was shifted in favor of Treg cells and away from cytotoxic cells (Clayton et al. 2007). Exosomes from nasopharyngeal carcinoma recruited Treg cells into the tumor through the chemokine CCL20, and mediated the conversion of the conventional T cells into Treg cells (Mrizak et al. 2014).

Under the influence of exosomes secreted by nasopharyngeal carcinoma cells, T-cell proliferation was 
Table 1 Summary of immunosuppressive effects elicited by exosomes

\begin{tabular}{|c|c|c|c|}
\hline Source of exosomes & Molecule & Effect on immune cells & References \\
\hline Jurkat and Raji cell lines & NKG2D ligands & Decoy for NKG2D receptor function & Hedlund et al. (2011) \\
\hline $\begin{array}{l}\text { Mesothelioma and various cancer cell } \\
\text { lines }\end{array}$ & TGF- $\beta$ & NKG2D down-modulation & Clayton et al. (2008) \\
\hline $\begin{array}{l}\text { Head and neck squamous cell carcinoma; } \\
\text { melanoma cell lines }\end{array}$ & FasL & $\begin{array}{l}\text { Promotion of Treg cell expansion and the } \\
\text { demise of anti-tumor CD } 8^{+} \text {effector T } \\
\text { cells, induction of TGF- } \beta \text { production } \\
\text { by Treg }\end{array}$ & Wieckowski et al. (2009) \\
\hline Colorectal cancer & FasL, TNF- $\alpha$ & $\mathrm{CD}^{+} \mathrm{T}$-cell apoptosis & Abusamra et al. (2005) \\
\hline Ovarian cancer & FasL & $\begin{array}{l}\text { Apoptosis and caspase- } 3 \text { activation } \\
\text { within T cells }\end{array}$ & Taylor et al. (2003) \\
\hline Melanoma & FasL & Apoptosis in lymphoid cells & Andreola et al. (2002) \\
\hline Colorectal cancer & FasL, TNF- $\alpha$ & T-cell apoptosis & Huber et al. (2005) \\
\hline B-cell lymphoma & FasL & T-cell apoptosis & Klinker et al. (2014) \\
\hline EBV-associated NPC & Galectin-9 & Apoptosis in EBV-specific $\mathrm{CD}^{+}{ }^{+}$cells & Klibi et al. (2009) \\
\hline Acute myeloid leukemia & Membrane-associated TGF- $\beta$ & Suppression of NK-cell function & Szczepanski et al. (2011) \\
\hline Various cancer cell lines & $\mathrm{CD} 39$ and $\mathrm{CD} 73$ & Generation of extracellular adenosine & Clayton et al. (2011) \\
\hline Pancreatic cancer & $\mathrm{Nd}$ & $\begin{array}{l}\text { Increased levels of } 9 \text { miRNAs, down- } \\
\text { regulation of }>200 \text { mRNAs }\end{array}$ & Ding et al. (2015) \\
\hline Lung carcinoma & $\begin{array}{l}\text { miR-23a } \\
\text { TGF- } \beta\end{array}$ & Inhibition of NK-cell function & Berchem et al. (2015) \\
\hline Melanoma & $\mathrm{Nd}$ & $\begin{array}{l}\text { TCR zeta-chain inactivation through } \\
\text { ROS }\end{array}$ & Söderberg et al. (2007) \\
\hline Head and neck cancer cell line & $\mathrm{Nd}$ & $\begin{array}{l}\text { Regulation of immune response-related } \\
\text { genes in T cells, up-regulation of TGF- } \\
\beta, \text { IL-10, COX-2, CD } 39, \text { CD73 and } \\
\text { adenosine production }\end{array}$ & Muller et al. (2016) \\
\hline Pancreatic cancer & miR-203 & $\begin{array}{l}\text { Down-regulation of TLR4 and down- } \\
\text { stream cytokines in DCs }\end{array}$ & Zhou et al. (2014) \\
\hline Nasopharyngeal carcinoma & $\begin{array}{l}\text { miR-24-3p, miR-891a, } \\
\text { miR-106a-5p, miR-20a-5p, } \\
\text { miR-1908 }\end{array}$ & $\begin{array}{l}\text { T-cell dysfunction through down-regu- } \\
\text { lation of the MAPK1 and JAK/STAT } \\
\text { pathways }\end{array}$ & Ye et al. (2014) \\
\hline $\begin{array}{l}\text { Lewis lung carcinoma cell line and } \\
\text { human embryonic kidney cell line }\end{array}$ & $\operatorname{miR}-214$ & $\begin{array}{l}\text { Down-regulation of PTEN and promotion } \\
\text { of Treg expansion }\end{array}$ & Yin et al. (2014) \\
\hline $\begin{array}{l}\text { Mesothelioma and various cancer cell } \\
\text { lines }\end{array}$ & TGF- $\beta$ & Induction of human Treg cells & Clayton et al. (2007) \\
\hline Nasopharyngeal carcinoma & $\mathrm{Nd}$ & $\begin{array}{l}\text { Conversion of the conventional } \mathrm{T} \text { cells } \\
\text { into Treg }\end{array}$ & $\begin{array}{l}\text { Mrizak et al. (2014) } \\
\text { Ye et al. (2014) }\end{array}$ \\
\hline Colorectal cancer & TGF- $\beta$ & Induction of Treg cells & Yamada et al. (2016) \\
\hline Melanoma & $\mathrm{Nd}$ & $\begin{array}{l}\text { Generation of CD14 }{ }^{+} \mathrm{HLA}-\mathrm{DR}^{-/ \mathrm{low}} \text { cells } \\
\text { secreting TGF- } \beta\end{array}$ & Valenti et al. (2006) \\
\hline Murine mammary adenocarcinoma & $\mathrm{Nd}$ & $\begin{array}{l}\text { Blockage of myeloid precursor differen- } \\
\text { tiation into DCs }\end{array}$ & Yu et al. (2007) \\
\hline B16 mouse model for human melanoma & $\mathrm{Nd}$ & MDSC inducion involving MyD88 & Liu et al. (2010) \\
\hline Mammary carcinoma & TGF- $\beta$, PgE2 & Promotion of MDSC differentiation & Xiang et al. (2009) \\
\hline Multiple myeloma & $\mathrm{Nd}$ & $\begin{array}{l}\text { Promotion of MDSC viability and pro- } \\
\text { liferation }\end{array}$ & Wang et al. (2016) \\
\hline Renal cancer & Hsp70 & $\begin{array}{l}\text { TLR2 mediated Stat } 3 \text { activation in } \\
\text { MDSC }\end{array}$ & $\begin{array}{l}\text { Diao et al. (2015) } \\
\text { Xiang et al. (2010) }\end{array}$ \\
\hline Various cancer cell lines & Hsp72 & $\begin{array}{l}\text { Stat } 3 \text { activation and IL- } 6 \text { production in } \\
\text { MDSC }\end{array}$ & Chalmin et al. (2010) \\
\hline Murine thymoma & $\mathrm{Nd}$ & $\begin{array}{l}\text { Induction of B cells with inhibitory } \\
\text { function }\end{array}$ & Yang et al. (2012a) \\
\hline Esophageal cancer & $\mathrm{Nd}$ & $\begin{array}{l}\text { Induction of regulatory B cells express- } \\
\text { ing TGF- } \beta\end{array}$ & Li et al. (2015) \\
\hline
\end{tabular}


Table 1 (continued)

\begin{tabular}{llll}
\hline Source of exosomes & Molecule & Effect on immune cells & References \\
\hline Ovarian cancer & miR-222 & $\begin{array}{c}\text { Conversion of M1 macrophages into the } \\
\text { M2 phenotype }\end{array}$ & Ying et al. (2016) \\
\hline
\end{tabular}

$N d$ not defined, EBV Epstein-Barr virus, NPC nasopharyngeal carcinoma, ROS reactive oxygen species, PTEN phosphatase and tensin homolog

inhibited, while Treg induction was stimulated (Ye et al. 2014). Furthermore, the production of IL-2, IL-17, and IFN- $\gamma$ was decreased indicating impaired immune stimulation. Extracellular vesicles from colorectal cancer cells activated Smad signaling in T cells through exosomal TGF- $\beta 1$ changing the phenotype into Treg-like cells (Yamada et al. 2016). In addition, miRNAs transported via microvesicles participated in the induction of the Treg cell phenotype, as shown for MiR-214 which mediated reduction of the PTEN (phosphatase and tensin homolog) level in mouse peripheral CD4 ${ }^{+} \mathrm{T}$ cells (Yin et al. 2014). Interestingly, exosomes were described to elicit antigen-specific immunosuppression (Yang et al. 2011, 2012b). The application of tumorderived exosomes suppressed a delayed-type hypersensitivity response to a model antigen in an antigen-specific manner. The exact mechanism is not known but might include modulation of APCs.

Tumor-derived vesicles are able to impair DC development and to induce MDSCs (Valenti et al. 2006). The presence of cancer exosomes severely impaired the differentiation of DCs from murine bone marrow precursors or from human monocytes (Yu et al. 2007). The induction of IL-6 expression in the precursor cells was partially responsible for the observed block in DC differentiation. Valenti et al. (2006) showed that tumor-derived vesicles not only inhibited DC differentiation, but actively skewed precursors toward the acquisition of a MDSC phenotype. These cells mediated negative regulation of effector cells, e.g., through the secretion of soluble TGF- $\beta$ (Valenti et al. 2006). Exosomes derived from murine breast carcinomas triggered the MDSC differentiation pathway, and this activity was dependent on prostaglandin E2 (PgE2) and TGF- $\beta$ (Xiang et al. 2009). In addition, exosomes released by human multiple myeloma cells promoted the viability and proliferation of MDSCs (Wang et al. 2016). MDSC survival was supported by the activation of Stat3 (Wang et al. 2015). Renal cancer cell-derived exosomes induced the phosphorylation of Stat 3 in MDSCs in a TLR2-dependent manner through the transfer of heatshock protein 70 (Hsp70) (Diao et al. 2015). Blocking the Hsp70/TLR2 interaction with a peptide aptamer reduced the ability of tumor-derived exosomes to stimulate MDSC activation (Gobbo et al. 2015). The dependence of MDSC expansion on TLR2 was further investigated and confirmed by Xiang et al. (2010). In addition, membrane-bound Hsp72 in exosomes derived from human and murine cancer cell lines activated MDSCs and stimulated their suppressive function via Stat3 activation and IL-6 production (Chalmin et al. 2010). The involvement of MyD88 in the recruitment and activity of MDSC after exposure of bone marrow derived cells to tumor exosomes was shown in mice (Liu et al. 2010). MyD88 is a downstream effector of TLR signaling, and thus the findings corroborate the critical involvement of the TLR pathway.

In addition, the promotion of $\mathrm{B}$ cells with inhibitory activity by cancer exosomes was reported (Yang et al. 2012a). Mycoplasma-infected murine thymoma and melanoma cells released exosomes that induced IL-10 production in splenic B cells. Another study described how under the influence of esophageal cancer-derived microvesicles naïve $\mathrm{B}$ cells developed into immunosuppressive regulatory B cells expressing TGF- $\beta$ (Li et al. 2015).

The conversion of cancer-suppressive cells into supporters of tumor growth and survival by exosomes was described for macrophages. Macrophages are the most abundant immune cells within the tumor microenvironment. Macrophages can be polarized into a cancersuppressive M1 or a tumor supportive M2 phenotype. Exosomes from epithelial ovarian cancer were shown to shift macrophages towards the M2 phenotype (Ying et al. 2016). The involvement of miR-222 transferred by the exosomes was proposed through down-regulation of SOCS3. A similar activity was attributed to miR-494 that inhibited macrophage polarization and switched them towards the immunosuppressive M2 type (Zhao et al. 2016). In a co-culture system of murine cell lines, pancreatic cancer cell-derived exosomes shifted macrophage polarization to the M2 phenotype ( $\mathrm{Su}$ et al. 2016). Overexpression of miR-155 and miR-125b-2 in the cancer cells reverted this effect and resulted in M1 polarized macrophages upon exosome exposure. The differentiation of monocytes into macrophages in the presence of colon cancer cell-derived EVs revealed increased IL-10 secretion and a mixed M1/M2 polarization status which, after longer incubation time, switched to the regulatory M2 phenotype (Baj-Krzyworzeka et al. 2016).

The great variety of mechanisms to induce immunosuppressive cells exemplifies the potential of EVs to modulate the function of recipient cells by the transfer of bioactive molecules. 
Exosomes Derived from Cancer Cells and Normal Cells Share Immune Signaling Functions

Recently, a very comprehensive review of the physiological roles of EVs was published covering their currently known functions in healthy organisms (Yáñez-Mó et al. 2015). This overview confirmed the crucial importance of EVs in intercellular signal transduction with effects on coagulation and angiogenesis, reproduction, embryonic development, tissue repair, organ homeostasis, and immunity. Communication between immune cells is one of the best characterized roles of exosomes and other EVs. Increased release of exosomes was observed upon interaction of DCs or B cells with T cells (Buschow et al. 2009; Muntasell et al. 2007), or when T-cell antigen receptors were engaged (Blanchard et al. 2002).

EVs from different sources exert immunosuppressive effects. Exosomes released from CD4-positive Th cells could suppress the activity of cytotoxic $\mathrm{T}$ cells (Zhang et al. 2011). Tolerogenic EVs derived from non-malignant cells contribute to the establishment and maintenance of the immune-privileged status of certain tissues. An important example is the human embryo which is protected during pregnancy from immune attacks by the exchange of EVs at the interface between the maternal placenta and the fetus. Placenta-derived exosomes were shown to suppress the immune system by carrying NKG2D ligands (MIC and the ULBP), which bind and down-regulate the NKG2D receptor on NK cells, $\mathrm{CD} 8^{+}$, and $\gamma \delta \mathrm{T}$ cells, consequently reducing the cytotoxicity of these cells (Hedlund et al. 2009; Mincheva-Nilsson et al. 2006). Furthermore, clusters of FasL and TRAIL were identified on placental exosomes able to trigger apoptosis in T cells (Stenqvist et al. 2013). MSCs are another source of tolerogenic EVs. It has been reported that the regenerative effects in tissue injury exerted by MSCs are mediated in part by EVs and this includes an immunosuppressive component consisting of both RNA and proteins (Arslan et al. 2013; Burrello et al. 2016; Cantaluppi et al. 2012).

The immunosuppressive effects of donor-derived exosomes were even used to prolong graft survival after transplantation. Heart allograft survival in MHC-mismatched rats could be prolonged by injection of exosomes derived from donor bone marrow DCs before transplantation (Pêche et al. 2006). In addition, in a mouse model, exosomes isolated from immature DCs in combination with immunosuppressive drugs improved cardiac allograft survival (Li et al. 2012).

Immunoregulatory functions have been identified for several miRNAs transferred by EVs from non-transformed cells. Alexander et al. (2015) reported that exosomes can modulate the response to endotoxin-induced inflammation by transferring miRNA to antigen presenting cells. Two
miRNAs that regulate inflammation, miR-146a and miR155, were released from DCs within EVs and were taken up by recipient DCs. Injection of miR-146a-containing exosomes into mice inhibited the inflammatory response to endotoxin. A role of miR-146a in modulation of adaptive immunity was also suggested by Curtale et al. (2010). Up-regulation of miR-146a in T cells after stimulation of the TCR resulted in an anti-apoptotic signal counteracting activation-induced cell death. In exosomes derived from Foxp $3^{+}$Treg cells, let-7d was found to suppress Th1 cell proliferation and IFN- $\gamma$ secretion (Okoye et al. 2014). A soluble T-cell suppressor factor recognized earlier to mediate antigen-specific inhibition of contact sensitivity was identified as miR-150. This miRNA was transported by exosomes derived from suppressor $\mathrm{T}$ cells (Bryniarski et al. 2013; Ptak et al. 2015) and the suppressive effect was dependent on the presence of macrophages (Nazimek et al. 2015).

As it is the case with all other mechanisms that support tumor growth, survival, and progression, also immunosuppression is not specific for cancer, but is abused during the disease to escape the immune surveillance program of the host.

\section{Conclusion}

Tumors are heterogeneous, and different cells within the tumor may use different immune-escape mechanisms, such as apoptosis induction, impaired antigen presentation, or secretion of immunosuppressive factors. Moreover, multiple mechanisms may develop in a single tumor cell. Therefore, it is questionable whether a single, predominant immune-escape mechanism can be identified in a tumor. Exosomes participate in all kinds of mechanisms by which cancer evades immune surveillance and takes control over the immune system. Several aspects of the in vivo activity of exosomes are still unknown, especially how far they spread from the site of secretion and what quantities are secreted and captured by target cells. However, it is clear that cancer-derived exosomes are able to induce alterations of immune cell functions and a deeper insight into the cellular and molecular mechanisms underlying tumor immune escape using exosomes may finally lead to novel therapeutic approaches for the benefit of cancer patients.

Acknowledgements The work was supported by Grant No. 2012/05/B/NZ2/00574 from the National Science Centre (NCN), Poland.

Open Access This article is distributed under the terms of the Creative Commons Attribution 4.0 International License (http:// creativecommons.org/licenses/by/4.0/), which permits unrestricted use, distribution, and reproduction in any medium, provided you give 
appropriate credit to the original author(s) and the source, provide a link to the Creative Commons license, and indicate if changes were made.

\section{References}

Abusamra AJ, Zhong Z, Zheng X et al (2005) Tumor exosomes expressing Fas ligand mediate $\mathrm{CD} 8^{+} \mathrm{T}$-cell apoptosis. Blood Cells Mol Dis 35:169-173

Alexander M, Hu R, Runtsch MC et al (2015) Exosome-delivered microRNAs modulate the inflammatory response to endotoxin. Nat Commun 6:7321

Al-Nedawi K, Meehan B, Micallef J et al (2008) Intercellular transfer of the oncogenic receptor EGFRvIII by microvesicles derived from tumour cells. Nat Cell Biol 10:619-624

Al-Nedawi K, Meehan B, Kerbel RS et al (2009) Endothelial expression of autocrine VEGF upon the uptake of tumor-derived microvesicles containing oncogenic EGFR. Proc Natl Acad Sci USA 106:3794-3799

André F, Chaput N, Schartz NE et al (2004) Exosomes as potent cellfree peptide-based vaccine. I. Dendritic cell-derived exosomes transfer functional MHC class I/peptide complexes to dendritic cells. J Immunol 172:2126-2136

Andreola G, Rivoltini L, Castelli C et al (2002) Induction of lymphocyte apoptosis by tumor cell secretion of FasL-bearing microvesicles. J Exp Med 195:1303-1316

Arslan F, Lai RC, Smeets MB et al (2013) Mesenchymal stem cellderived exosomes increase ATP levels, decrease oxidative stress and activate PI3K/Akt pathway to enhance myocardial viability and prevent adverse remodeling after myocardial ischemia/reperfusion injury. Stem Cell Res 10:301-312

Aubertin K, Silva AK, Luciani N et al (2016) Massive release of extracellular vesicles from cancer cells after photodynamic treatment or chemotherapy. Sci Rep 6:35376

Baietti MF, Zhang Z, Mortier E et al (2012) Syndecan-syntenin-ALIX regulates the biogenesis of exosomes. Nat Cell Biol 14:677-685

Baj-Krzyworzeka M, Mytar B, Szatanek R et al (2016) Colorectal cancer-derived microvesicles modulate differentiation of human monocytes to macrophages. J Transl Med 14:36

Benitez R, Godelaine D, Lopez-Nevot MA et al (1998) Mutations of the beta2-microglobulin gene result in a lack of HLA class I molecules on melanoma cells of two patients immunized with MAGE peptides. Tissue Antigens 52:520-529

Berchem G, Noman MZ, Bosseler M et al (2015) Hypoxic tumorderived microvesicles negatively regulate NK cell function by a mechanism involving TGF- $\beta$ and miR23a transfer. Oncoimmunology 5:e1062968

Blanchard N, Lankar D, Faure F et al (2002) TCR activation of human $\mathrm{T}$ cells induces the production of exosomes bearing the TCR/CD3/zeta complex. J Immunol 168:3235-3241

Bogen B (1996) Peripheral T cell tolerance as a tumor escape mechanism: deletion of $\mathrm{CD}^{+}{ }^{+} \mathrm{T}$ cells specific for a monoclonal immunoglobulin idiotype secreted by a plasmacytoma. Eur J Immunol 26:2671-2679

Bolukbasi MF, Mizrak A, Ozdener GB et al (2012) miR-1289 and "Zipcode"-like Sequence Enrich mRNAs in microvesicles. Mol Ther Nucleic Acids 1:e10

Bryniarski K, Ptak W, Jayakumar A et al (2013) Antigen-specific, antibody-coated, exosome-like nanovesicles deliver suppressor T-cell microRNA-150 to effector T cells to inhibit contact sensitivity. J Allergy Clin Immunol 132:170-181

Bu N, Wu H, Sun B et al (2011) Exosome-loaded dendritic cells elicit tumor-specific $\mathrm{CD}^{+}$cytotoxic $\mathrm{T}$ cells in patients with glioma. $\mathrm{J}$ Neurooncol 104:659-667
Burrello J, Monticone S, Gai C et al (2016) Stem Cell-Derived Extracellular Vesicles and Immune-Modulation. Front Cell. Dev Biol 4:83

Buschow SI, Nolte-'t Hoen EN, van Niel G et al (2009) MHC II in dendritic cells is targeted to lysosomes or $\mathrm{T}$ cell-induced exosomes via distinct multivesicular body pathways. Traffic 10:1528-1542

Cantaluppi V, Gatti S, Medica D et al (2012) Microvesicles derived from endothelial progenitor cells protect the kidney from ischemia-reperfusion injury by microRNA-dependent reprogramming of resident renal cells. Kidney Int 82:412-427

Chalmin F, Ladoire S, Mignot G et al (2010) Membrane-associated Hsp72 from tumor-derived exosomes mediates STAT3dependent immunosuppressive function of mouse and human myeloid-derived suppressor cells. J Clin Invest 120:457-471

Chen W, Wang J, Shao C et al (2006) Efficient induction of antitumor $\mathrm{T}$ cell immunity by exosomes derived from heat-shocked lymphoma cells. Eur J Immunol 36:1598-1607

Chow A, Zhou W, Liu L et al (2014) Macrophage immunomodulation by breast cancer-derived exosomes requires Toll-like receptor 2-mediated activation of NF- $\mathrm{KB}$. Sci Rep 4:5750

Clayton A, Mitchell JP, Court J et al (2007) Human tumor-derived exosomes selectively impair lymphocyte responses to interleukin-2. Cancer Res 67:7458-7466

Clayton A, Mitchell JP, Court J et al (2008) Human tumor-derived exosomes down-modulate NKG2D expression. J Immunol 180:7249-7258

Clayton A, Al-Taei S, Webber J et al (2011) Cancer exosomes express CD39 and CD73, which suppress $\mathrm{T}$ cells through adenosine production. J Immunol 187:676-683

Colombo M, Moita C, van Niel G et al (2013) Analysis of ESCRT functions in exosome biogenesis, composition and secretion highlights the heterogeneity of extracellular vesicles. J Cell Sci 126(Pt 24):5553-5565

Colombo M, Raposo G, Théry C (2014) Biogenesis, secretion, and intercellular interactions of exosomes and other extracellular vesicles. Annu Rev Cell Dev Biol 30:255-289

Curtale G, Citarella F, Carissimi C et al (2010) An emerging player in the adaptive immune response: microRNA-146a is a modulator of IL-2 expression and activation-induced cell death in T lymphocytes. Blood 115:265-273

Dai S, Wei D, Wu Z et al (2008) Phase I clinical trial of autologous ascites-derived exosomes combined with GM-CSF for colorectal cancer. Mol Ther 16:782-790

Damo M, Wilson DS, Simeoni E et al (2015) TLR-3 stimulation improves anti-tumor immunity elicited by dendritic cell exosome-based vaccines in a murine model of melanoma. Sci Rep 5:17622

Denzer K, van Eijk M, Kleijmeer MJ et al (2000) Follicular dendritic cells carry MHC class II-expressing microvesicles at their surface. J Immunol 165:1259-1265

Diao J, Yang X, Song X et al (2015) Exosomal Hsp70 mediates immunosuppressive activity of the myeloid-derived suppressor cells via phosphorylation of Stat3. Med Oncol 32:453

Ding G, Zhou L, Qian Y et al (2015) Pancreatic cancerderived exosomes transfer miRNAs to dendritic cells and inhibit RFXAP expression via miR-212-3p. Oncotarget 6:29877-29888

Escudier B, Dorval T, Chaput N et al (2005) Vaccination of metastatic melanoma patients with autologous dendritic cell (DC) derived-exosomes: results of the first phase I clinical trial. J Transl Med 3:10

Finn OJ (2012) Immuno-oncology: understanding the function and dysfunction of the immune system in cancer. Ann Oncol 23(Suppl 8):viii6-viii9 
Garrido F, Ruiz-Cabello F, Cabrera T et al (1997) Implications for immunosurveillance of altered HLA class I phenotypes in human tumours. Immunol Today 18:89-95

Gasparoto TH, de Souza Malaspina TS, Benevides L et al (2010) Patients with oral squamous cell carcinoma are characterized by increased frequency of suppressive regulatory $\mathrm{T}$ cells in the blood and tumor microenvironment. Cancer Immunol Immunother 59:819-828

Gehrmann U, Hiltbrunner S, Georgoudaki AM et al (2013) Synergistic induction of adaptive antitumor immunity by co-delivery of antigen with $\alpha$-galactosylceramide on exosomes. Cancer Res 73:3865-3876

Gibbings DJ, Ciaudo C, Erhardt M et al (2009) Multivesicular bodies associate with components of miRNA effector complexes and modulate miRNA activity. Nat Cell Biol 11:1143-1149

Gobbo J, Marcion G, Cordonnier M et al (2015) Restoring anticancer immune response by targeting tumor-derived exosomes with a HSP70 peptide aptamer. J Natl Cancer Inst 108:djv330

Gould GW, Lippincott-Schwartz J (2009) New roles for endosomes: from vesicular carriers to multi-purpose platforms. Nat Rev Mol Cell Biol 10:287-292

Groh V, Wu J, Yee C et al (2002) Tumour-derived soluble MIC ligands impair expression of NKG2D and T-cell activation. Nature 419:734-738

Gu X, Erb U, Büchler MW et al (2015) Improved vaccine efficacy of tumor exosome compared to tumor lysate loaded dendritic cells in mice. Int J Cancer 136:E74-E84

Hao S, Bai O, Li F et al (2007) Mature dendritic cells pulsed with exosomes stimulate efficient cytotoxic T-lymphocyte responses and anti-tumour immunity. Immunology 120:90-102

Hedlund M, Stenqvist AC, Nagaeva O et al (2009) Human placenta expresses and secretes NKG2D ligands via exosomes that down-modulate the cognate receptor expression: evidence for immunosuppressive function. J Immunol 183:340-351

Hedlund M, Nagaeva O, Kargl D et al (2011) Thermal- and oxidative stress causes enhanced release of NKG2D ligand-bearing immunosuppressive exosomes in leukemia/lymphoma $\mathrm{T}$ and $\mathrm{B}$ cells. PLoS One 6:e16899

Hellwinkel JE, Redzic JS, Harland TA et al (2016) Glioma-derived extracellular vesicles selectively suppress immune responses. Neuro Oncol 18:497-506

Hicklin DJ, Marincola FM, Ferrone S (1999) HLA class I antigen down-regulation in human cancers: T-cell immunotherapy revives an old story. Mol Med Today 5:178-186

Hong CS, Muller L, Boyiadzis M et al (2014) Isolation and characterization of $\mathrm{CD}_{3} 4^{+}$blast-derived exosomes in acute myeloid leukemia. PLoS One 9:e103310

Huber V, Fais S, Iero M et al (2005) Human colorectal cancer cells induce T-cell death through release of proapoptotic microvesicles: role in immune escape. Gastroenterology 128:1796-1804

Huotari J, Helenius A (2011) Endosome maturation. EMBO J 30:3481-3500

Jaiswal JK, Andrews NW, Simon SM (2002) Membrane proximal lysosomes are the major vesicles responsible for calcium-dependent exocytosis in non-secretory cells. J Cell Biol 159:625-635

Johnsen AK, Templeton DJ, Sy M et al (1999) Deficiency of transporter for antigen presentation (TAP) in tumor cells allows evasion of immune surveillance and increases tumorigenesis. $\mathbf{J}$ Immunol 163:4224-4231

Johnstone RM, Adam M, Hammond JR et al (1987) Vesicle formation during reticulocyte maturation. Association of plasma membrane activities with released vesicles (exosomes). J Biol Chem 262:9412-9420

Kahlert C, Melo SA, Protopopov A et al (2014) Identification of double-stranded genomic DNA spanning all chromosomes with mutated KRAS and p53 DNA in the serum exosomes of patients with pancreatic cancer. J Biol Chem 289:3869-3875

Kalra H, Simpson RJ, Ji H et al (2012) Vesiclepedia: a compendium for extracellular vesicles with continuous community annotation. PLoS Biol 10:e1001450

Kindt TJ, Goldsby RA, Osborne BA et al (2007) Immunology. WH Freeman \& Co Ltd, New York

Klibi J, Niki T, Riedel A et al (2009) Blood diffusion and Th1-suppressive effects of galectin-9-containing exosomes released by Epstein-Barr virus-infected nasopharyngeal carcinoma cells. Blood 113:1957-1966

Klinker MW, Lizzio V, Reed TJ et al (2014) Human B cell-derived lymphoblastoid cell lines constitutively produce fas ligand and secrete $\mathrm{MHCII}(+) \mathrm{FasL}(+)$ killer exosomes. Front Immunol $5: 144$

Koppers-Lalic D, Hackenberg M, Bijnsdorp IV et al (2014) Nontemplated nucleotide additions distinguish the small RNA composition in cells from exosomes. Cell Rep 8:1649-1658

Kosaka N, Iguchi H, Hagiwara K et al (2013) Neutral sphingomyelinase 2 (nSMase2)-dependent exosomal transfer of angiogenic microRNAs regulate cancer cell metastasis. J Biol Chem 288:10849-10859

Kowal J, Tkach M, Théry C (2014) Biogenesis and secretion of exosomes. Curr Opin Cell Biol 29:116-125

Lemmon SK, Traub LM (2000) Sorting in the endosomal system in yeast and animal cells. Curr Opin Cell Biol 12:457-466

Li X, Li JJ, Yang JY et al (2012) Tolerance induction by exosomes from immature dendritic cells and rapamycin in a mouse cardiac allograft model. PLoS One 7:e44045

Li W, Mu D, Tian F et al (2013) Exosomes derived from Rab27aoverexpressing tumor cells elicit efficient induction of antitumor immunity. Mol Med Rep 8:1876-1882

Li Y, An J, Huang S et al (2015) Esophageal cancer-derived microvesicles induce regulatory B cells. Cell Biochem Funct 33:308-313

Li X, Wang S, Zhu R et al (2016) Lung tumor exosomes induce a proinflammatory phenotype in mesenchymal stem cells via NFKBTLR signaling pathway. J Hematol Oncol 9:42

Lind MH, Rozell B, Wallin RP et al (2004) Tumor necrosis factor receptor 1-mediated signaling is required for skin cancer development induced by NF-kappaB inhibition. Proc Natl Acad Sci USA 101:4972-4977

Liu Y, Xiang X, Zhuang X et al (2010) Contribution of MyD88 to the tumor exosome-mediated induction of myeloid derived suppressor cells. Am J Pathol 176:2490-2499

Liu Y, Lai L, Chen Q et al (2012) MicroRNA-494 is required for the accumulation and functions of tumor-expanded myeloidderived suppressor cells via targeting of PTEN. J Immunol 188:5500-5510

Liu ZM, Wang YB, Yuan XH (2013) Exosomes from murine-derived GL26 cells promote glioblastoma tumor growth by reducing number and function of CD8 + T cells. Asian Pac J Cancer Prev 14:309-314

Ljunggren HG, Kärre K (1990) In search of the 'missing self': MHC molecules and NK cell recognition. Immunol Today $11: 237-244$

Lundholm M, Schröder M, Nagaeva O et al (2014) Prostate tumorderived exosomes down-regulate NKG2D expression on natural killer cells and $\mathrm{CD} 8^{+} \mathrm{T}$ cells: mechanism of immune evasion. PLoS One 9:e108925

Luzio JP, Pryor PR, Bright NA (2007) Lysosomes: fusion and function. Nat Rev Mol Cell Biol 8:622-632

Maeda H, Shiraishi A (1996) TGF-beta contributes to the shift toward Th2-typeresponses through direct and IL-10-mediated pathways in tumor-bearing mice. J Immunol 156:73-78

Maeurer MJ, Gollin SM, Martin D et al (1996) Tumor escape from immune recognition: lethal recurrent melanoma in a patient 
associated with downregulation of the peptide transporter protein TAP-1 and loss of expression of the immunodominant MART-1/Melan-A antigen. J Clin Invest 98:1633-1641

Mahaweni NM, Kaijen-Lambers ME, Dekkers J et al (2013) Tumour-derived exosomes as antigen delivery carriers in dendritic cell-based immunotherapy for malignant mesothelioma. J Extracell Vesicles 2:22492

Marton A, Vizler C, Kusz E et al (2012) Melanoma cell-derived exosomes alter macrophage and dendritic cell functions in vitro. Immunol Lett 148:34-38

Mathivanan S, Ji H, Simpson RJ (2010) Exosomes: extracellular organelles important in intercellular communication. J Proteomics 73:1907-1920

Matsuda M, Salazar F, Petersson M et al (1994) Interleukin 10 pretreatment protects target cells from tumor- and allo-specific cytotoxic T cells and down-regulates HLA class I expression. J Exp Med 180:2371-2376

Miller IV, Grunewald TG (2015) Tumour-derived exosomes: tiny envelopes for big stories. Biol Cell 107:287-305

Mincheva-Nilsson L, Nagaeva O, Chen T et al (2006) Placentaderived soluble MHC class I chain-related molecules down-regulate NKG2D receptor on peripheral blood mononuclear cells during human pregnancy: a possible novel immune escape mechanism for fetal survival. J Immunol 176:3585-3592

Möbius W, Ohno-Iwashita Y, van Donselaar EG et al (2002) Immunoelectron microscopic localization of cholesterol using biotinylated and non-cytolytic perfringolysin O. J Histochem Cytochem 50:43-55

Morelli AE, Larregina AT, Shufesky WJ et al (2004) Endocytosis, intracellular sorting, and processing of exosomes by dendritic cells. Blood 104:3257-3266

Morishita M, Takahashi Y, Matsumoto A et al (2016) Exosome-based tumor antigens-adjuvant co-delivery utilizing genetically engineered tumor cell-derived exosomes with immunostimulatory CpG DNA. Biomaterials 111:55-65

Mrizak D, Martin N, Barjon C et al (2014) Effect of nasopharyngeal carcinoma-derived exosomes on human regulatory $\mathrm{T}$ cells. $\mathrm{J}$ Natl Cancer Inst 107:363

Muller L, Mitsuhashi M, Simms P et al (2016) Tumor-derived exosomes regulate expression of immune function-related genes in human T cell subsets. Sci Rep 6:20254

Muntasell A, Berger AC, Roche PA (2007) T cell-induced secretion of MHC class II-peptide complexes on B cell exosomes. EMBO J 26:4263-4272

Muralidharan-Chari V, Clancy J, Plou C et al (2009) ARF6-regulated shedding of tumor cell-derived plasma membrane microvesicles. Curr Biol 19:1875-1885

Näslund TI, Gehrmann U, Qazi KR et al (2013) Dendritic cell-derived exosomes need to activate both $\mathrm{T}$ and $\mathrm{B}$ cells to induce antitumor immunity. J Immunol 190:2712-2719

Nazimek K, Ptak W, Nowak B et al (2015) Macrophages play an essential role in antigen-specific immune suppression mediated by $\mathrm{T} \mathrm{CD}^{+}$cell-derived exosomes. Immunology 146:23-32

Ohm JE, Gabrilovich DI, Sempowski GD et al (2003) VEGF inhibits T-cell development and may contribute to tumor-induced immune suppression. Blood 101:4878-4886

Okoye IS, Coomes SM, Pelly V et al (2014) MicroRNA-containing T-regulatory-cell-derived exosomes suppress pathogenic $\mathrm{T}$ helper 1 cells. Immunity 41:89-103

Pan BT, Johnstone R (1984) Selective externalization of the transferrin receptor by sheep reticulocytes in vitro. Response to ligands and inhibitors of endocytosis. J Biol Chem 259:9776-9782

Pan BT, Teng K, Wu C et al (1985) Electron microscopic evidence for externalization of the transferrin receptor in vesicular form in sheep reticulocytes. J Cell Biol 101:942-948
Pap E, Pállinger E, Falus A (2011) The role of membrane vesicles in tumorigenesis. Crit Rev Oncol Hematol 79:213-223

Pasche B (2001) Role of transforming growth factor beta in cancer. J Cell Physiol 186:153-168

Pêche H, Renaudin K, Beriou G et al (2006) Induction of tolerance by exosomes and short-term immunosuppression in a fully MHC-mismatched rat cardiac allograft model. Am J Transplant 6:1541-1550

Ptak W, Nazimek K, Askenase PW et al (2015) From mysterious supernatant entity to miRNA-150 in Antigen-specific exosomes: a history of hapten-specific T suppressor factor. Arch Immunol Ther Exp 63:345-356

Rao Q, Zuo B, Lu Z et al (2016) Tumor-derived exosomes elicit tumor suppression in murine hepatocellular carcinoma models and humans in vitro. Hepatology 64:456-472

Raposo G, Nijman HW, Stoorvogel W et al (1996) B lymphocytes secrete antigen-presenting vesicles. J Exp Med 183:1161-1172

Rappa G, Mercapide J, Anzanello F et al (2013) Biochemical and biological characterization of exosomes containing prominin-1/ CD133. Mol Cancer 12:62

Raulet DH, Guerra N (2009) Oncogenic stress sensed by the immune system: role of natural killer cell receptors. Nat Rev Immunol 9:568-580

Restifo NP, Esquivel F, Kawakami Y et al (1993) Identification of human cancers deficient in antigen processing. J Exp Med 177:265-272

Rong L, Li R, Li S et al (2016) Immunosuppression of breast cancer cells mediated by transforming growth factor- $\beta$ in exosomes from cancer cells. Oncol Lett 11:500-504

Rotem-Yehudar R, Groettrup M, Soza A et al (1996) LMP-associated proteolytic activities and TAP-dependent peptide transport for class $1 \mathrm{MHC}$ molecules are suppressed in cell lines transformed by the highly oncogenic adenovirus 12. J Exp Med 183:499-514

Rountree RB, Mandl SJ, Nachtwey JM et al (2011) Exosome targeting of tumor antigens expressed by cancer vaccines can improve antigen immunogenicity and therapeutic efficacy. Cancer Res 71:5235-5244

Schuler PJ, Saze Z, Hong CS et al (2014) Human CD4 ${ }^{+}$CD $39^{+}$regulatory $\mathrm{T}$ cells produce adenosine upon co-expression of surface CD73 or contact with $\mathrm{CD} 73^{+}$exosomes or $\mathrm{CD} 73^{+}$cells. Clin Exp Immunol 177:531-543

Shevach EM (2002) $\mathrm{CD}^{+} \mathrm{CD} 25^{+}$suppressor T cells: more questions than answers. Nat Rev Immunol 2:389-400

Skog J, Würdinger T, van Rijn S et al (2008) Glioblastoma microvesicles transport RNA and proteins that promote tumour growth and provide diagnostic biomarkers. Nat Cell Biol 10:1470-1476

Smyth LA, Ratnasothy K, Tsang JY et al (2013) CD73 expression on extracellular vesicles derived from $\mathrm{CD} 4^{+} \mathrm{CD} 25^{+} \mathrm{Foxp}^{+} \mathrm{T}$ cells contributes to their regulatory function. Eur $\mathrm{J}$ Immunol 43:2430-2440

Söderberg A, Barral AM, Soderstrom M et al (2007) Redox-signaling transmitted in trans to neighboring cells by melanoma-derived TNF-containing exosomes. Free Radic Biol Med 43:90-99

Staveley-O'Carroll K, Sotomayor E, Montgomery J et al (1998) Induction of antigen-specific $\mathrm{T}$ cell anergy: an early event in the course of tumor progression. Proc Natl Acad Sci USA 95:1178-1183

Stenqvist AC, Nagaeva O, Baranov V et al (2013) Exosomes secreted by human placenta carry functional Fas ligand and TRAIL molecules and convey apoptosis in activated immune cells, suggesting exosome-mediated immune privilege of the fetus. J Immunol 191:5515-5523

Su MW, Yu SL, Lin WC et al (2016) Smoking-related microRNAs and mRNAs in human peripheral blood mononuclear cells. Toxicol Appl Pharmacol 305:169-175 
Szajnik M, Czystowska M, Szczepanski MJ et al (2010) Tumorderived microvesicles induce, expand and up-regulate biological activities of human regulatory $\mathrm{T}$ cells (Treg). PLoS One 5:e11469

Szczepanski MJ, Szajnik M, Welsh A et al (2011) Blast-derived microvesicles in sera from patients with acute myeloid leukemia suppress natural killer cell function via membraneassociated transforming growth factor-beta1. Haematologica 96:1302-1309

Taylor DD, Gercel-Taylor C, Lyons KS et al (2003) T-cell apoptosis and suppression of T-cell receptor/CD3-zeta by Fas ligandcontaining membrane vesicles shed from ovarian tumors. Clin Cancer Res 9:5113-5119

Thakur BK, Zhang H, Becker A et al (2014) Double-stranded DNA in exosomes: a novel biomarker in cancer detection. Cell Res 24:766-769

Théry C, Duban L, Segura E et al (2002) Indirect activation of naïve $\mathrm{CD} 4^{+} \mathrm{T}$ cells by dendritic cell-derived exosomes. Nat Immunol 3:1156-1162

Théry C, Ostrowski M, Segura E (2009) Membrane vesicles as conveyors of immune responses. Nat Rev Immunol 9:581-593

Trajkovic K, Hsu C, Chiantia S et al (2008) Ceramide triggers budding of exosome vesicles into multivesicular endosomes. Science 319:1244-1247

Valadi H, Ekström K, Bossios A et al (2007) Exosome-mediated transfer of mRNAs and microRNAs is a novel mechanism of genetic exchange between cells. Nat Cell Biol 9:654-659

Valenti R, Huber V, Filipazzi P et al (2006) Human tumor-released microvesicles promote the differentiation of myeloid cells with transforming growth factor-beta-mediated suppressive activity on T lymphocytes. Cancer Res 66:9290-9298

Verma M, Lam TK, Hebert E et al (2015) Extracellular vesicles: potential applications in cancer diagnosis, prognosis, and epidemiology. BMC Clin Pathol 15:6

Viaud S, Théry C, Ploix S et al (2010) Dendritic cell-derived exosomes for cancer immunotherapy: what's next? Cancer Res 70:1281-1285

Villarroya-Beltri C, Gutiérrez-Vázquez C, Sánchez-Cabo F et al (2013) Sumoylated hnRNPA2B1 controls the sorting of miRNAs into exosomes through binding to specific motifs. Nat Commun 4:2980

Vinay DS, Ryan EP, Pawelec G et al (2015) Immune evasion in cancer: mechanistic basis and therapeutic strategies. Semin Cancer Biol 35(Suppl):S185-S198

Wang J, De Veirman K, De Beule N et al (2015) The bone marrow microenvironment enhances multiple myeloma progression by exosome-mediated activation of myeloid-derived suppressor cells. Oncotarget 6:43992-44004

Wang J, De Veirman K, Faict S et al (2016) Multiple myeloma exosomes establish a favourable bone marrow microenvironment with enhanced angiogenesis and immunosuppression. J Pathol 239:162-173

Webber JP, Spary LK, Sanders AJ et al (2015) Differentiation of tumour-promoting stromal myofibroblasts by cancer exosomes. Oncogene 34:290-302

Wieckowski EU, Visus C, Szajnik M et al (2009) Tumor-derived microvesicles promote regulatory $\mathrm{T}$ cell expansion and induce apoptosis in tumor-reactive activated CD8C T lymphocytes. J Immunol 183:3720-3730

Wolfers J, Lozier A, Raposo G et al (2001) Tumor-derived exosomes are a source of shared tumor rejection antigens for CTL crosspriming. Nat Med 7:297-303
Wu L, Zhang X, Zhang B et al (2016) Exosomes derived from gastric cancer cells activate NF- $\mathrm{KB}$ pathway in macrophages to promote cancer progression. Tumour Biol 37:12169-12180

Xiang X, Poliakov A, Liu C et al (2009) Induction of myeloidderived suppressor cells by tumor exosomes. Int $\mathrm{J}$ Cancer 124:2621-2633

Xiang X, Liu Y, Zhuang X et al (2010) TLR2-mediated expansion of MDSCs is dependent on the source of tumor exosomes. Am J Pathol 177:1606-1610

Xie Y, Bai O, Zhang $\mathrm{H}$ et al (2010) Tumor necrosis factor gene-engineered J558 tumor cell-released exosomes stimulate tumor antigen P1A-specific CD8 ${ }^{+}$CTL responses and antitumor immunity. Cancer Biother Radiopharm 25:21-28

Yamada N, Kuranaga Y, Kumazaki M et al (2016) Colorectal cancer cell-derived extracellular vesicles induce phenotypic alteration of $T$ cells into tumor-growth supporting cells with transforming growth factor- $\beta 1$-mediated suppression. Oncotarget 7:27033-27043

Yáñez-Mó M, Siljander PR, Andreu Z et al (2015) Biological properties of extracellular vesicles and their physiological functions. J Extracell Vesicles 4:27066

Yang C, Kim SH, Bianco NR et al (2011) Tumor-derived exosomes confer antigen-specific immunosuppression in a murine delayed-type hypersensitivity model. PLoS One 6:e22517

Yang C, Chalasani G, Ng YH et al (2012a) Exosomes released from Mycoplasma infected tumor cells activate inhibitory B cells. PLoS One 7:e36138

Yang C, Ruffner MA, Kim SH et al (2012b) Plasma-derived MHC class $\mathrm{II}^{+}$exosomes from tumor-bearing mice suppress tumor antigen-specific immune responses. Eur J Immunol 42:1778-1784

Yao Y, Chen L, Wei W et al (2013) Tumor cell-derived exosometargeted dendritic cells stimulate stronger $\mathrm{CD} 8^{+} \mathrm{CTL}$ responses and antitumor immunities. Biochem Biophys Res Commun 436:60-65

Ye SB, Li ZL, Luo DH et al (2014) Tumor-derived exosomes promote tumor progression and T-cell dysfunction through the regulation of enriched exosomal microRNAs in human nasopharyngeal carcinoma. Oncotarget 5:5439-5452

Yin Y, Cai X, Chen X et al (2014) Tumor-secreted miR-214 induces regulatory T cells: a major link between immune evasion and tumor growth. Cell Res 24:1164-1180

Ying X, Wu Q, Wu X et al (2016) Epithelial ovarian cancer-secreted exosomal miR-222-3p induces polarization of tumor-associated macrophages. Oncotarget 7:43076-43087

Yokokawa J, Cereda V, Remondo C et al (2008) Enhanced functionality of $\mathrm{CD}^{+} \mathrm{CD} 25$ (high)FoxP3 ${ }^{+}$regulatory $\mathrm{T}$ cells in the peripheral blood of patients with prostate cancer. Clin Cancer Res 14:1032-1040

Yu S, Liu C, Su K et al (2007) Tumor exosomes inhibit differentiation of bone marrow dendritic cells. J Immunol 178:6867-6875

Zhang H, Xie Y, Li W et al (2011) CD4(+) T cell-released exosomes inhibit CD8(+) cytotoxic T-lymphocyte responses and antitumor immunity. Cell Mol Immunol 8:23-30

Zhang B, Yin Y, Lai RC et al (2014) Immunotherapeutic potential of extracellular vesicles. Front Immunol 5:518

Zhao G, Miao H, Li X et al (2016) TGF- $\beta 3$-induced miR-494 inhibits macrophage polarization via suppressing PGE2 secretion in mesenchymal stem cells. FEBS Lett 590:1602-1613

Zhou M, Chen J, Zhou L et al (2014) Pancreatic cancer derived exosomes regulate the expression of TLR4 in dendritic cells via miR-203. Cell Immunol 292:65-69 